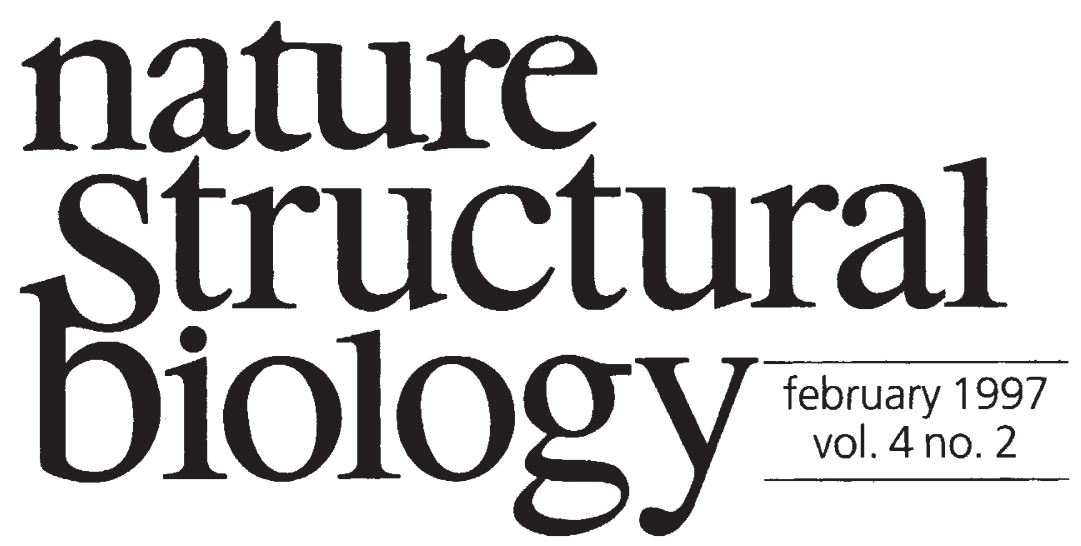

\title{
Fault lines in the HIV core?
}

The biological role of cyclophilin A (CypA) - and the cyclophilins in general-remains somewhat elusive, even now some 13 years after their discovery. Their role in immunosuppression is well documented but this is, of course, a gain-of-function effect that has little or nothing to do with CypA's normal cellular function. A recent observation may shed light on the role of CypA: it is both packaged into HIV-1 particles and plays an important, but unknown role in the viral life cycle. Further insight into this intriguing association is provided by the recent report of the structure of the complex between the N-terminal domain of the HIV-1 capsid protein (CA) and human cyclophilin $\mathrm{A}^{1}$.

That the cyclophilins play a fundamental role in cellular metabolism is suggested by their conservation across all phyla and their presence in different subcellular compartments. CypA has peptide prolyl isomerase (PPIase, or rotamerase) activity in vitro, catalysing the cis-trans interconversion of the Xaa-Pro peptide bond. It is presumed that the enzymic function of the protein relates to its function in vivo-suggesting that cyclophilins are involved in protein folding. Indeed, CypA can enhance the rate of folding (by up to $\sim 300$-fold) in vitro of a wide range of proline-containing proteins.

Another view would have it that the that the main function of CypA is to act as a mediator of specific protein-protein interactions ${ }^{2}$, in a manner similar to Src homology domains 2 and 3. For example, it is suggested that the NinaA protein, an integral membrane cyclophilin in Drosophila, may function as

Fig. 1 The cycolphilin-capsid protein complex. Cyclophilin (upper left) interacts with the sequence $\mathrm{PX}_{4} \mathrm{PX}_{2} \mathrm{P}$ (brown) on a loop connecting helices 4 (blue) and 5 (purple) of the CA structure. The $\mathrm{N}$-terminal Pro 1 residue (centre right), which anchors one end of the $\beta$-hairpin/sheet (red), forms a salt bridge with Asp 55 at the C-terminal end of helix 2 (yellow). This interaction is only possible after cleavage of CA from the Gag polyprotein 1,7. The high conservation of both these residues suggests they play an important role in CA function ${ }^{1,7}$ (see text). Figure reproduced from ref. 1 with permission. a chaperone (as opposed to a PPIase) specifically for the visual pigment rhodopsin, aiding its passage through the secretory system ${ }^{3}$. The PPIase activity of the cyclophilins would then, it is argued, simply be a side-effect of the ability to bind to proline residues and sequester them in a hydrophobic environment (the active site), thereby lowering the local dielectric constant and favouring the isomerisation reaction ${ }^{2}$.

What of CypA in HIV-1 particles? The protein is packaged into the virus through its association with the Gag polypro- 


\section{IMAGE \\ UNAVAILABLE FOR COPYRIGHT REASONS}

Fig. 2 Strips of CA molecules running through the crystal. The four central CA molecules are colour coded as in Fig. 1. CA-CA interfaces are denoted by ' 1 ' and ' 2 ' and yellow arrows and are different from those reported in ref. 8. Figure reproduced from ref. 1 with permission.

tein and specifically with one of the three major polypeptide chains derived from $\mathrm{Gag}$, the capsid protein $(\mathrm{CA})^{4-6}$. CA is responsible for, among other things, forming the conical-shaped core of the virion. The CypA binding site on CA has been mapped to a small region of CA that contains a cluster of proline residues- $\mathrm{PX}_{4} \mathrm{PX}_{2} \mathrm{PX}_{5} \mathrm{P}-$ conserved in all $\mathrm{HIV}-1$ isolates (but not in other primate immunodeficiency viruses $)^{5}$. Mutation of the second (bold) proline in this sequence disrupts cycolphilin binding to CA, its incorporation into the virion and an early step in viral replication (as does treatment with cyclosporin A $)^{5,6}$. Particles lacking CypA have a normal morphology, indicating that CypA is not required for the Gag-directed particle formation.

The recent structure determination ${ }^{1}$ reveals that the CA-CypA interface is formed exclusively by a portion of the conserved CA sequence- $\mathrm{PX}_{4} \mathrm{PX}_{2} \mathrm{P}$, found on a loop between helices 4 and 5 (Fig. 1) -which binds in the active site of CypA. The peptide bond of the central (bold) proline is in the trans, rather than the cis conformation seen in all previous CypA-ligand complexes. This unusual conformation pushes the preceding Ala and Gly residues (AlaGlyPro) deep into the active site cavity allowing them, and surrounding residues, to form a series of sequence-specific interactions with CypA.

As the authors point out, the interaction provides a general mechanism by which CypA could mediate specific protein-protein interactions. The burial of these Ala and Gly residues in CA (and the formation of a specific interface) is only possible because of the presence of Gly-the absence of a side-chainbearing amino acid avoids a steric clash with the relatively rigid binding surface of CypA. In the case of relatively non-specific interactions (required for CypA's more general, and presently assumed role as a PPIase in protein folding) the presence of a non-glycine (side-chain bearing) amino acid directly $\mathrm{N}$ terminal to Pro (XaaPro) would, because of steric interference, prevent the formation of a highly specific interface. This, in turn, would result in distortion of the ligand as it bound and this, it is suggested, would drive the isomerization reaction.

Intriguingly, the CA molecules are lined up in isolated strips that stretch the entire length of the crystal (Fig. 2). This, the authors speculate, may reflect in part the organisation of $\mathrm{CA}$ in the two-dimensional surface of viral core. One part of the CA-CA interface-the N-terminal B-hairpin-can only form after proteolytic cleavage of the Gag polyprotein to yield CA (Fig. 1) ${ }^{1,7}$. This cleavage-induced conformational change, which extends the CA interface, could

1. Gamble, T.R. et al., Cell 87, 1285-1294 (1996).

2. Schreiber, S.L. \& Crabtree, G.R. Immunology Today 13, 136-142 (1992).

3. Baker, E.K., Colley, N.J. \& Zuker, C.S. EMBO J. 13, 488-4895 (1994).

4. Luban, J., Bossolt, K.a.,Franke,E.K., Kalpana, G.V. \& Goff, S.P. Cell, 73, 1067-1078 (1993).

5. Franke, E.K., Yuan, H.E.H. \& Luban J. Nature 372, 359-362 (1994)

6. Thali, M.et al. Nature 372, 363-365 (1994).

7. Gitti, R.K. et al. Science 273, 231-235 (1996).

8. Momany, C. et al. Nature Struct. Biol, 3, 763770 (1996). therefore trigger 'maturation', the process whereby monomeric CA coalesces into the cone-shaped core ${ }^{1,7}$. Gamble and colleagues also note that the complex provides an explanation for the role of CypA in disassembly of the core. The individual CA strips are kept apart through steric interference caused by the CypA molecules bound along their edges. In the virion, the lower ratio of CypA to CA (1:10, rather than 1:1 in the CypA-CA complex) would likely still be sufficient to disrupt the two-dimensional array of CA molecules, causing the core to fall apart. 